\title{
A development optical course based on optical fiber white light interference
}

Haili Jiang, Qiuhua Sun, Yancheng Zhao, Qingbo Li

Haili Jiang, Qiuhua Sun, Yancheng Zhao, Qingbo Li, "A development optical course based on optical fiber white light interference," Proc. SPIE 10452, 14th Conference on Education and Training in Optics and Photonics: ETOP 2017, 104526U (16 August 2017); doi: 10.1117/12.2270719

SPIE Event: 14th Conference on Education and Training in Optics and Photonics, ETOP 2017, 2017, Hangzhou, China 


\title{
A development optical course based on optical fiber white light interference
}

\author{
Jiang Haili, Sun Qiuhua, Zhao Yancheng, Li Qingbo \\ (Harbin Engineering University, College of science, Heilongjiang, Harbin, 150001)
}

\begin{abstract}
The Michelson interferometer is a very important instrument in optical part for college physics teaching. But most students only know the instrument itself and don't know how to use it in practical engineering problems. A case about optical fiber white light interference based on engineering practice was introduced in the optical teaching of college physics and then designed a development course of university physical optics part. This system based on low-coherence white light interferometric technology can be used to measure distribution strain or temperature. It also could be used in the case of temperature compensation mode.This teaching design can use the

knowledge transfer rule to enable students to apply the basic knowledge in the university physics to the new knowledge domain, which can promote the students' ability of using scientific methods to solve complex engineering problems.
\end{abstract}

Keywords: White light interference; Learning transfer; Michelson interferometer; College Physics

\section{Introduction}

In our institutions of higher education system, university physics is a required course, it is not only for students to follow professional knowledge learning and lay a solid foundation of physics, but also plays a positive role in cultivating students' ability to solve practical problems. Since the introduction of the authentication method of international higher engineering education in China from the beginning of 2006, "engineering education accreditation standards" "graduation requirements" clearly pointed out: the graduates to mathematics, natural science, engineering foundation and professional knowledge to solve complex engineering problems ${ }^{[1-3]}$. From the point of view of scientific thinking and scientific research ability, physics is the most scientific training course. The teaching content of college physics of each chapter is a set of independent scientific content, each chapter can give students the extension field and expand, so the course of university physics than other courses more suitable for cultivating students' scientific research ability.

Although the college physics curriculum has so richly endowed by nature advantages, but the teachers in teaching found basic knowledge learned in college physics teaching is not obvious in the professional knowledge and the effect of forward along the extension of the field. For most college students, professional knowledge is more important than basic knowledge, professional knowledge to be more able to solve practical engineering problems than the basic knowledge; so many students pay more attention to the application of professional knowledge and skills of learning and ignore the course of university physics learning ${ }^{[4-6]}$.

In order to solve the above problems, the most effective way is to learn the knowledge in the practical application environment, in order to make the knowledge to return to practice, in order to realize the transfer

14th Conference on Education and Training in Optics and Photonics: ETOP 2017, edited by Xu Liu,

Xi-Cheng Zhang, Proc. of SPIE Vol. 10452, 104526U · ( 2017 ICO, IEEE, OSA, SPIE

CCC code: $0277-786 \mathrm{X} / 17 / \$ 18 \cdot$ doi: $10.1117 / 12.2270719$

Proc. of SPIE Vol. $10452104526 \mathrm{U}-1$ 
of knowledge. Therefore, how to further knowledge into college physics teaching, how the various new technologies to expand the teaching content of college physics, how to design research teaching of university physics in the college physics knowledge to solve a key ${ }^{[7-9]}$ migration, it is difficult to improve the students' ability to solve practical problems.

This paper from the research field: optical fiber sensor of optical fiber white light interference system is designed to expand the contents of college physics of wave optics, make students realize the basic knowledge we have learned more practical significance, so that students from undergraduate begin with the most basic understanding of scientific research, make students realize that even science the research frontier also cannot do without our basic knowledge, to stimulate students to university physics as a basic subject of attention and study enthusiasm.

\section{The teaching design based on learning transfer}

Learning transfer plays an important role in the transfer of basic knowledge to applied knowledge and engineering practice ${ }^{[7-8]}$. According to the existing knowledge system and whether or not to establish a link between the knowledge of the classification of learning, learning can be divided into meaningful learning and mechanical learning two. Meaningful learning refers to a meaningful learning process learners can establish non-human substantial contact learned in the new knowledge system and knowledge system, which is to have the knowledge to new knowledge transfer system. Meaningful learning is more important for the effectiveness of knowledge transfer.

In this course, the physical model of Michelson interferometer is chosen as the starting point of the knowledge of "College Physics". Then from the Michelson interferometer into ordinary optical fiber in the optical fiber by Michelson interferometer based on extended to optical fiber white light interference system and its measurement should be of practical engineering parameters of stress and strain and temperature, finally realizes the transfer optical knowledge base to practical engineering problems. Specific curriculum design flow chart was shown in figure 1.

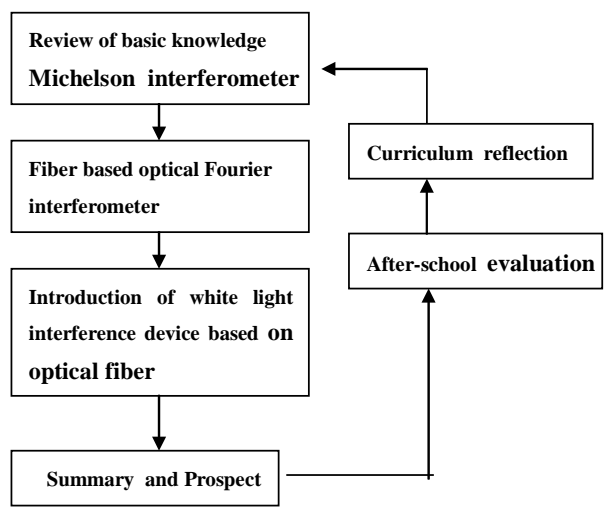

Fig. 1 The flow chart of class design 


\section{The implementation of classroom teaching based on white light interference}

3.1 The review Michelson interferometer

Firstly we review the physics model of the Michelson interferometer. Michelson interferometer is a kind of instrument which uses the principle of interference to measure precisely. The main idea is that the incident light can be divided into two coherent beams by using a half anti lens, and then the two coherent beams can be met. The basic device is shown in figure 2 .

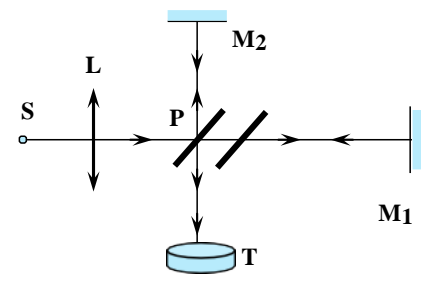

Fig. 2 The basic device of Michelson interferometer

\subsection{Fiber Michelson interferometer}

The next step is to guide the students to use the optical fiber to convert the traditional Michelson interferometer into an optical fiber based Michelson interferometer, as shown in fig3. Device of fiber Michelson interferometer is mainly composed of LED light source, $2 * 2$ coupler, a scanning mirror, etc. The light path 1 incident into from an optical fiber. The light path 1 split into light path 2 and 3 after $2 * 2$ coupler. The light path 2 was reflected into reflective light path $2^{\prime} \quad$ via coated optical fiber reflective end; The light path 3 was reflected from optical scanning mirror and formed light path ${ }^{3}$. The light path $2^{\prime}$ and light path ${ }^{3}$ meet together after $2 * 2$ coupler and interfered. The interference fringes can be observed from the detector.

Through the transformation of the traditional Michelson interferometer, so that students have a preliminary understanding of the fiber, but also expand students' thinking, stimulate students' creative thinking and interest in learning, positive thinking of other devices can also be achieved by Michelson interferometer.

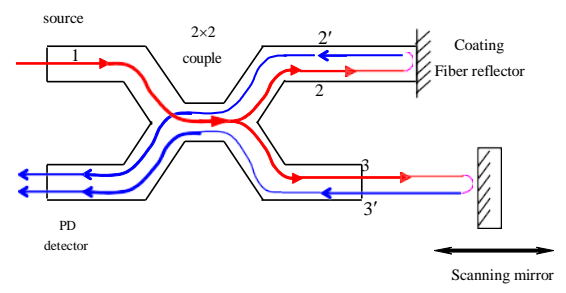

Fig. 3 Michelson interferometer based on optical fiber 
3.3 White light interferometric system based on optical fiber

Next, the existing physical knowledge to further knowledge of the new migration, to explain to students on the basis of optical fiber white light interference system and the basic principles of devices. The using of wide spectrum light source can realize absolute measurement of displacement. The monochromatic (coherent length) light interferometer can only achieve corresponding measure radian phase range of optical path difference; interferometer output will vary periodically, so the measurement of absolute displacement can not. If the light source was replaced with a broadband light source, because of its coherent length is very small, the main interference fringe signals in central fringe position can be accurately determined, so as to realize the so-called absolute measurement, and has high measurement precision.

As shown in Figure 3, the light source uses a wide spectrum light source, the light path 2 is used as the sensing arm, and the light path 3 is used as the reference arm, and the optical path difference between the sensing arm and the reference arm can be changed by a scanning mirror at the end of the optical path 3 . When the reference path between the arm and the sensing arm two beams is less than the coherence length, it will generate a white light interferogram. When the optical path of the two beams is absolutely equal, the optical path is exactly matched, and the interference pattern in the detector will have a central stripe with the maximum amplitude.

When the sensing arm is subjected to external strain, the optical path changes, and the change of the optical path of the sensor can be obtained by measuring the position of the mirror corresponding to the zero level central fringe, as shown in figure 4. So it can realize the absolute measurement of the physical quantities such as temperature and so on.

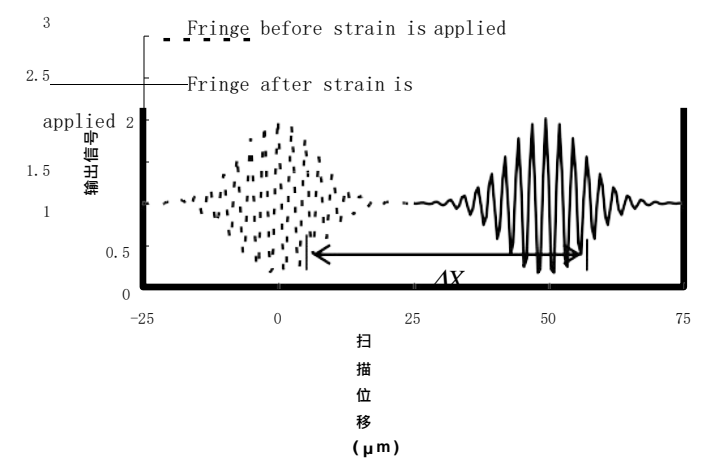

Fig. 4 shift of the central fringe of the scanning mirror

At this point, the basic knowledge of this section of the Michelson interferometer to knowledge transfer about optical fiber based white light interference system of is over. At the end of the course, teachers summarize the new knowledge in this section, and inspire students to do more in-depth research. 


\section{Conclusion}

Although the university physics is a relatively broad and old knowledge structure, and seems like far away from modern physics. But as long as the teachers do more effort to concentrate on studying, will be able to integrate the knowledge and science through classical physics. This way not only makes the physics course can get rid of the old, dull color, but also wearing a novel and lively aura; also to fully mobilize the enthusiasm of students, to cultivate the spirit of scientific research from their undergraduate students, deepen the importance in learning physics, improved the ability of students to solve complex engineering problems.

\section{Reference}

[1]Haili Jiang, Yonggui Yuan, Jun Yang, Libo Yuan . A Novel Multi-path Combination Matching Michelson Interferometer for Strainldeformation Sensing, Advanced Sensor Systems and Applications IV,2010.10.18.

[2]Haili Jiang, Yonggui Yuan, Jun Yang, Libo Yuan . The theoretical and experimental study on white light interferometric fiber optic sensors network based on Michelson and Mach-Zehnder interferometers , 2011 Academic International Symposium on Optoelectronics and Microelectronics Technology, AISOMT 2011, 2011.10.

[3] Mayer , R. E. Teaching of subject matter [J]. Annu. Rev. Psychol. 2004 , $55: 715$ - 741

[4] Ehri L. C. ,Nunes S. R. ,Simone R. ,Willows D. M. et al. Phonemic awareness inst ruction helps children learn to read: evidence from the National Reading Panel's meta - analysis [J] . Read. Res. Q. ,2001, 36 :250 - 287

[5] Mayer R. E. Thinking ,Problem Solving, Cognition[M] . New York : Freeman. 2nd ed. 1992. [6] WANG Ruimin. College physics teaching design of exemplifying Hopfield neural network model[J].College Physics,2014(4):44-48.

[7] GOU Lidan,ZHANG Zhiying, ZHU Ruihan. An approximate solution of two dimensional coupling nonlinear harmonic oscillator[J]. College Physics,2012(8):39-43

[8] LI Chuang, LIU Zhaoji, WU Bing, et al. High-precision optical fiber end microfabrication system and the experimental research[J].Applied Science and Technology,2013,40(2):31-34.

[9] MA Kun, WU Haijun, YANG Jiyong, et al. A fiber seismograph demodulation method based on COSTAS loop[J]. Applied Science and Technology,2016,43(6):67-71. 